\title{
Prior-Based Automatic Segmentation of the Carotid Artery Lumen in TOF MRA (PASCAL)
}

\author{
Jana Hutter ${ }^{1,2}$, Hannes G. Hofmann ${ }^{1}$, Robert Grimm ${ }^{1}$, Andreas Greiser ${ }^{3}$, \\ Marc Saake $^{4}$, Joachim Hornegger ${ }^{1,2}$, Arnd Dörfler ${ }^{4}$, and Peter Schmitt ${ }^{3}$ \\ 1 Pattern Recognition Lab, University Erlangen-Nuremberg, Germany \\ 2 School of Advanced Optical Technologies, Erlangen, Germany \\ 3 Siemens Healthcare, Magnetic Resonance, Erlangen, Germany \\ ${ }^{4}$ Department of Neuroradiology, University Erlangen-Nuremberg, Germany
}

\begin{abstract}
In current clinical practice, examinations of the carotid artery bifurcation are commonly carried out with Computed Tomography Angiography (CTA) or contrast-enhanced Magnetic Resonance Angiography (ce MRA). Quantitative information about vessel morphology, extracted from segmentations, is promising for diagnosis of vessel pathologies. However, both above-mentioned techniques require the administration of contrast media. In contrary, non-ce MRA methods such as Time-of-Flight (TOF) provide fully non-invasive imaging without any exogenous contrast agent. The diagnostic value of TOF MRA, however, for assessment of the carotid bifurcation area can be hampered due to its susceptibility to irregular blood flow patterns. Conventional methods for lumen segmentation are very sensitive to such signal voids and produce inaccurate results. In this work, a novel, fully automatic 3D segmentation algorithm is proposed which uses prior knowledge about irregular flow patterns. The presented technique has been successfully tested on eleven volunteer datasets as well as in a patient case, offering the comparison to CTA images. The sensitivity could be increased by $29.2 \%$ to $85.6 \%$ compared to standard level set methods. The root mean squared error in diameter measurements was reduced from $4.85 \mathrm{~mm}$ to $1.44 \mathrm{~mm}$.
\end{abstract}

\section{Introduction}

Stroke is one of the world leading causes of premature death [1] and shows increasing incidence numbers. Fast and reliable diagnosis of its cause is essential for treatment decisions. A common stroke source is stenosis in the internal carotid artery (ICA). Although CTA and Digital Subtraction Angiography (DSA) are still the gold standard for the carotid arteries, the risk for complications [2] and the exposition to ionizing radiation lead to a growing use of MRA. Besides the already widely used contrast-enhanced MR techniques, non-ce MR methods such as TOF allow contrast-agent-free, non-invasive imaging.

TOF relies on the inflow of blood perpendicular to the imaging plane to generate contrast between the vessel lumen and surrounding static tissue. Signal 


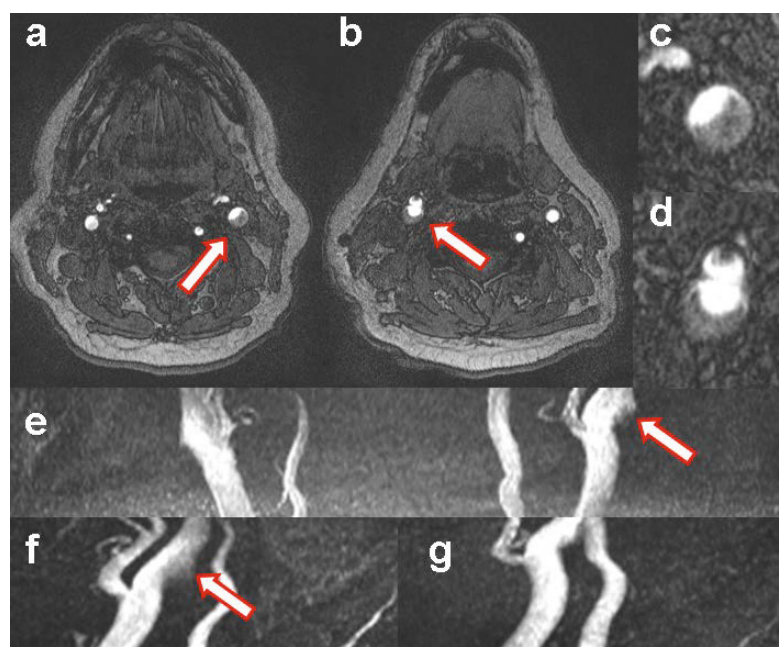

Fig. 1. Representative TOF dataset: (a) (b) Axial slices showing signal voids (indicated by arrows). (c) (d) Zoomed views. (e) Coronal MIP, (f),(g) sagittal MIPs.

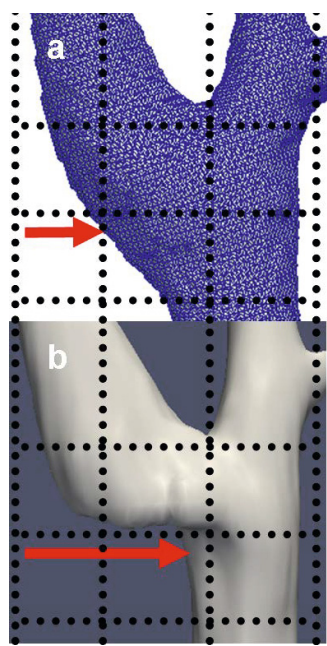

Fig. 2. (a) CTA and (b) MRA segmentation of the same patient

loss is observed in regions with irregular, fluctuating or reverse flow, which leads to intravoxel signal dephasing. In Fig. 1, typical TOF data of the carotids shows the characteristic artifacts pointed at by arrows. This susceptibility to irregular flow patterns [1] limits the usage for diagnosis as stenoses cannot be robustly identified. In critical regions, the lumen can show lower intensity close to the noise level, and strong signal gradients may occur inside. Though the true vessel delineation is still recognizable for the trained human eye, both effects constitute major problems for classical segmentation approaches as the sharp signal borders can easily be misinterpreted as the vessel wall. Especially the widely used Maximum Intensity Projection (MIP) visualization (Fig.1(e)-(g)) suffers from the weak differentiation between the vessel signal and the surrounding signal.

Existing approaches can roughly be divided in stochastic and level-set or active contour based methods. There are algorithms specifically designed for nonce MRA [3 - 8] as well as some focusing on the carotid arteries [3], [6], 9 -12]. But only very few methods [3], [6] address the specific problem of visualizing the challenging bifurcation region in non-ce MRA.

In this work, we propose a method that accounts for the special properties of the MRA TOF acquisition and uses knowledge about regions prone to artifacts to identify even low intensity lumen regions. Contrary to most of the stated methods, our proposed method works fully automatically.

\section{Segmentation}

Comparison of the segmentations for both a CTA (Fig.2(a), done with ITKSNAP [13]) and a TOF (Fig.2(b), done with a standard level set approach) 


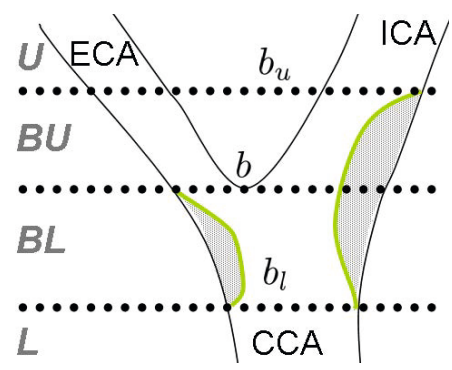

Fig. 3. Division of the ROI. Irregular flow zones are shaded

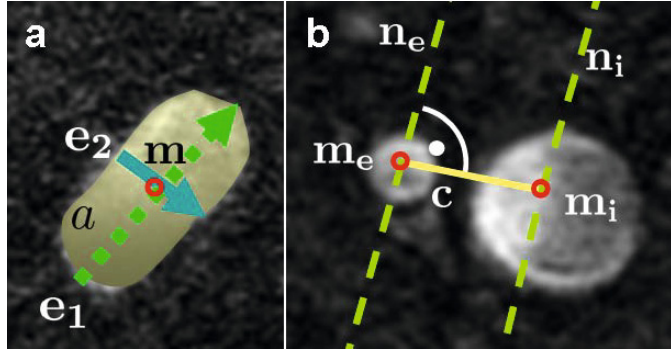

Fig. 4. (a) Ellipsoid fitting. (b) Detection of outer wall areas in BU.

scan reveals problems of conventional MRA TOF segmentation methods. Segmentation problems in the lower part of the ICA are indicated by arrows. Use of this segmentation for any quantitative evaluation would lead to significant underestimation of the vessel lumen. This problem is addressed by our fully automatic algorithm consisting of the following three steps.

\subsection{MIP Generation and Threshold-Based Pre-Segmentation}

In a first step, sagittal and transversal MIPs are generated from the slices of the 3D TOF scan. The regions of interest (ROI) are identified by adaptive thresholding. Noisy regions are eliminated as candidates by searching connected areas in slice direction. This leads to reduced computational effort as well as a more stable segmentation.

\subsection{Detection of the Vessel Tree Skeleton and the Bifurcation}

The common carotid artery (CCA) is differentiated from the vertebral artery in the first caudal slice based on the MIP pre-segmentation by size and connectivity to the bifurcation. The skeleton, consisting of center point localizations and bifurcation slices, is extracted as follows: The threshold result of the preceding slice $k-1$ is analyzed using ellipsoid fitting. This yields the major axis $\mathbf{e}_{\mathbf{1}-1, v}$, the minor axis $\mathbf{e}_{2 k-1, v}$, the area $a_{k-1, v}$ and the center $\mathbf{m}_{k-1, v}$ for each detected vessel segment $v$, where $v=0$ indicates the largest segment (see Fig.4(a)). Voxels on the major axis are used as seed points for the region-growing segmentation in slice $k$.

For each slice $f(k)=e(k)+a(k)$ is calculated and the bifurcation slice $b$ is determined as the slice with maximal value of $f(k)$. The ellipsoid-similarity measure $e(k)$ favors elongated ellopsoids, like those which occur just under the bifurcation. The area ratio $a(k)$ helps to differentiate the main ICA-ECA bifurcation, where both vessels have generally close area values, from small ascending branches:

$$
e(k)=\frac{\left\|\mathbf{e}_{1 k-1,0}\right\|}{\left\|\mathbf{e}_{2 k-1,0}\right\|} \quad \text { and } \quad a(k)=\frac{\min _{v} a_{k, v}}{\max _{v} a_{k, v}} .
$$


The extracted information is used to find regions that are potentially sensitive to flow artifacts. Theoretical and computational fluid dynamic studies show that these irregular flow zones are located at the outer walls at the origins of ICA and ECA [14], 15]] (Fig. 3, shaded regions).

Geometric Information. The volume is subdivided into four regions as illustrated in Fig. 3. The CCA part $\mathbf{L}$, the lower bifurcation part $\mathbf{B L}$ where the CCA has a fully ellipsoidal form, BU above the bifurcation, and the upper part $\mathbf{U}$. The distant parts $\mathbf{L}$ and $\mathbf{U}$ remain uncorrected, BL is fully corrected. Special attention is paid to $\mathbf{B U}$ where the region of the outer walls is critical. These regions are determined by identifying the outer halves of ICA and ECA. Therefore, their center points $\mathbf{m}_{i}$ and $\mathbf{m}_{e}$ (see Fig. 4(b)) are connected $\left(\mathbf{c}=\mathbf{m}_{i}-\mathbf{m}_{e}=\left(c_{1}, c_{2}\right)^{\mathrm{T}}\right)$ and the normal direction is computed as $\mathbf{n}=\left(-c_{2}, c_{1}\right)^{\mathrm{T}}=\left(n_{1}, n_{2}\right)^{\mathrm{T}}$. Then the normal equations through the center points $n_{i}(x): \mathbb{R} \mapsto \mathbb{R}$ and $n_{e}(x): \mathbb{R} \mapsto \mathbb{R}$ are

$$
n_{i}(x)=\frac{n_{1}}{n_{2}} x+m_{i, 2}-\frac{n_{1}}{n_{2}} m_{i, 1}, n_{e} \text { accordingly. }
$$

\subsection{Level Set Evolution Using Prior Information}

After these preprocessing steps, a full 3D level set evolution is applied. A contour of an open set $\omega$ is represented as the zero level of the higher dimensional scalar function $\Phi(\mathbf{x}): \mathbb{R}^{3} \mapsto \mathbb{R}$ with $\mathbf{x}=\left(x_{1}, x_{2}, x_{3}\right)^{\mathrm{T}} \in \mathbb{R}^{3} . \Phi(\mathbf{x})<0$ holds for points inside the borders. The distance regularized level set evolution as proposed by $\mathrm{Li}$ et al. [16] is used, which enforces the level set function to keep the desired shape by the distance regularization term without the need for re-initializations. Also, the $C^{1}$ approximation of the Heaviside function $H_{a p p}(\Phi)$ and the approximated Dirac function $\delta_{a p p}(\Phi)$ are used.

The speed term $F(\Phi)$ consists of the regularization term $R(\Phi)$ and an external energy term $E(\Phi, \mathbf{x})$ pushing the level set evolution in the desired direction based on the image intensities $I(\mathbf{x})$ :

$$
F(\Phi)=\mu R(\Phi)+E(\Phi, \mathbf{x})
$$

A standard method to minimize the functional $F$ is the steady state solution of the gradient flow which equals to

$$
\frac{\partial \Phi}{\partial t}=-\mu \frac{\partial R}{\partial \Phi}-\frac{\partial E}{\partial \Phi}
$$

The external energy term $E(\Phi, \mathbf{x})$ is composed of the edge term $F_{E}(\Phi)$, the area term $F_{A}(\Phi)$ and the additional prior knowledge term $F_{P}(\Phi, \mathbf{x})$ :

$$
E(\Phi, \mathbf{x})=\lambda F_{E}(\Phi)+\alpha F_{A}(\Phi)+\nu F_{P}(\Phi, \mathbf{x})
$$

where $\lambda, \alpha$ and $\nu>0$ are the coefficients regulating the weight of the corresponding terms. The prior knowledge term $F_{P}(\Phi, \mathbf{x})$ consists of intensity deviations 
from the mean background intensity level $u_{0}$, a weighting function $w(\mathbf{x})$ and the actual contour:

$$
F_{P}(\Phi, \mathbf{x})=\int_{\Omega} P(\mathbf{x}) H_{a p p}(-\Phi) d \mathbf{x}=\int_{\Omega}\left(I(\mathbf{x})-u_{0}\right)^{2} w(\mathbf{x}) H_{a p p}(-\Phi) d \mathbf{x} .
$$

A novel key element in PASCAL is the weighting term $w(\mathbf{x})$ which depends on the regions as explained in Fig. 3 and Sect.2.2. Contrary to approaches such as Scherl et al. [11], which uses integrated intensity deviations to differentate plaque from lumen in CTA images, extracted skeleton information and physical knowledge about flow irregularities is included with $w(\mathbf{x})$ :

$$
w(\mathbf{x})=\left\{\begin{array}{l}
0, \quad \text { if } x_{3}<b_{l}, \\
1, \quad \text { if } b_{l} \leq x_{3}<b, \\
\max \left(n_{i}\left(x_{1}\right)-x_{2}, 0\right) \cdot \max \left(x_{2}-n_{e}\left(x_{1}\right), 0\right), \quad \text { if } b \leq x_{3} \leq b_{u}, \\
0, \quad \text { if } x_{3}>b_{u} .
\end{array}\right.
$$

The most important case is $b \leq x_{3} \leq b_{u}$, which limits the regularization to the region close to the outer vessel wall just above the bifurcation by using the precalculated normals $n_{i}$ and $n_{e}$. Using the variational principle and the relation $\frac{\partial H_{a p p}}{\partial \Phi}=\delta_{a p p}$, the gradient descent flow equals to:

$$
\frac{\partial \Phi}{\partial t}=\mu \operatorname{div}\left(d_{p}\left(\|\nabla \Phi\|^{2}\right) \nabla \Phi\right)+\delta_{a p p}(\Phi(\mathbf{x}))\left(\lambda \operatorname{div}\left(g \frac{\nabla \Phi}{\|\nabla \Phi\|^{2}}\right)+\alpha g+\nu P(\mathbf{x})\right)
$$

where $g=1 /\left(1+\|\nabla I\|^{2}\right)$ and $d_{p}$ as described in [16]. The level set equation is spatially discretized by using central differences for the spatial derivatives and temporally using finite differences with a time step $\Delta t$. The pre-segmentation serves as initial value $\Phi^{0}(\mathbf{x})$.

\section{Experiments and Results}

\subsection{Experimental Setup}

TOF data was acquired in eleven volunteers (FOV $180 \mathrm{~mm} \times 180 \mathrm{~mm}$, imaging matrix $512 \times 512$, 3 slabs resulting in 51 slices, flip angle $25^{\circ}$, slice thickness $0.5 \mathrm{~mm}, \mathrm{TE}=3.76 \mathrm{~ms}, \mathrm{TR}=23 \mathrm{~ms}$ ) on a clinical scanner at $3 \mathrm{~T}$ (MAGNETOM Verio, Siemens Healthcare). Clinical data was acquired from a patient undergoing in addition a CTA examination (SOMATOM Definition AS+, Siemens Healthcare, rotation time $0.3 \mathrm{~s}$, increment $0.4 \mathrm{~mm}$, injection of $50 \mathrm{~mL}$ iodinated contrast agent, slice thickness $0.6 \mathrm{~mm}$, matrix size $512 \times 512,196$ slices, in-plane resolution $0.46 \mathrm{~mm} \times 0.46 \mathrm{~mm})$.

Two segmentations have been performed. The standard level set (SLS) segmentation corresponds to the method proposed by Li et al. [16] with the following parameters: $\lambda=20.0, \alpha=-10, \Delta t=0.2$ and $\mu=0.1$. The parameters for our proposed new method (PASCAL) have been identically chosen. The additional parameter $\nu$ was set to 0.08 . The same threshold initialization and the same number of iterations were used for both methods. 


\subsection{Quantitative Evaluation}

Segmentation Accuracy Study. For all twelve datasets, manual segmentations of a subset of slices were created by three independent readers including an experienced radiologist and MR scientists familiar with TOF data as gold standard. For the evaluation of the proposed segmentation method, sensitivity, specificity, accuracy, the positive predictive value (PPV) and the Dice coefficient were calculated both for the reference level set segmentation SLS and our novel PASCAL method. Only detail patches around the vessel have been used for this quantitative evaluation to ensure meaningful values especially for the specificity. Furthermore, five people manually detected the bifurcation point in the TOF image and the mean of the results was compared with the fully automatically detected positions.

Vessel Diameter. A clinical experiment was carried out with the acquired CTA and MRA patient dataset concentrating on quantitative measurements of the bifurcation and ICA diameter, which is used to quantify stenoses, for example with the NASCET criterion [2]. The diameter was measured at five positions in the bifurcation $\left(B_{1}-B_{5}\right)$ and three in the ICA $\left(I_{1}-I_{3}\right)$ both in the CTA dataset and the obtained MRA segmentation result.

\subsection{Results}

Segmentation results of the volunteer study are shown in Fig. 5 and Table 1 comparing a standard level set approach and the PASCAL method. Our approach significantly outperforms the conventional segmentation. In particular in the critical regions close to the bifurcation sensitivity values of $85.6 \%$ in comparison to $66.2 \%$ for the state of the art approach were achieved. The very high specificity of over $99 \%$ as well as a stable high PPV valueof above $97 \%$ for both approaches is particularly important to offer a reliable diagnosis of stenoses in this region. The proposed algorithm was furthermore able to detect the skeleton and the bifurcation region in all volunteer datasets. The root mean squared error between the automatically and manually detected positions was $0.4 \mathrm{~mm}$, which is below the slice thickness of the used data. The quantitative measurements in the clinical dataset (Table 2) yield diameters very close to the corresponding CTA measurements. The root mean squared error of the diameter was reduced from $4.85 \mathrm{~mm}$ to $1.44 \mathrm{~mm}$.

Table 1. Evaluation of the segmentation results in comparison the gold standard

\begin{tabular}{|c|c|c|c|c|c|c|c|c|c|c|}
\hline \multirow{2}{*}[\%]{} & \multicolumn{3}{|c|}{ Bifurcation region (BL, BU) } & \multicolumn{4}{c|}{ Distant regions (L, U) } \\
\cline { 2 - 11 } & Sens. & Spec. & Acc. & Dice & PPV & Sens. & Spec. & Acc. & Dice & PPV \\
\hline SLS & 66.2 & 99.8 & 95.7 & 78.8 & 98.7 & 69.9 & 99.9 & 97.8 & 81.8 & 99.3 \\
PASCAL & 85.6 & 99.6 & 97.8 & 90.7 & 97.1 & 79.5 & 99.8 & 98.5 & 87.8 & 98.4 \\
\hline
\end{tabular}




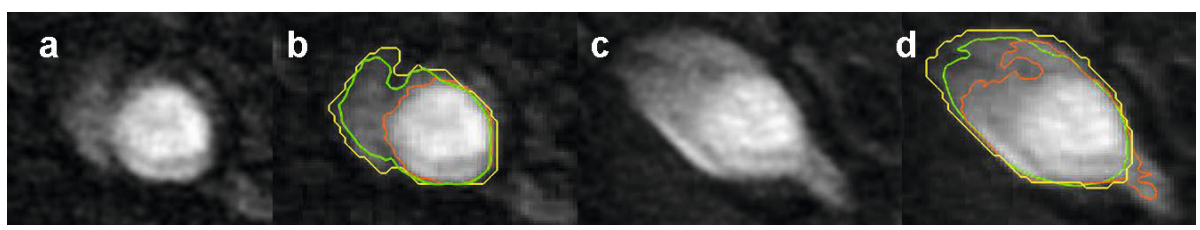

Fig. 5. (a) and (c) Two adjacent TOF slices. (b) and (d) Segmentation results. The yellow border demarcates the gold standard, the orange line the SLS and the green line shows the improved segmentation using PASCAL.

Table 2. Diameter of the carotis interna and the bifurcation in a clinical data set

\begin{tabular}{|cc||c|c|c|c|c||c|c|c|}
\hline & $B_{1}$ & $B_{2}$ & $B_{3}$ & $B_{4}$ & $B_{5}$ & $I_{1}$ & $I_{2}$ & $I_{3}$ \\
\hline CTA SLS $[\mathrm{mm}]$ & 18.5 & 15.5 & 13.8 & 11.3 & 9.0 & 8.3 & 8.4 & 7.7 \\
MRA SLS [mm] & 18.0 & 13.1 & 10.4 & 9.0 & 7.4 & 7.6 & 7.5 & 7.5 \\
MRA PASCAL [mm] & 18.1 & 14.9 & 13.2 & 11.0 & 8.1 & 8.1 & 7.9 & 7.8 \\
\hline
\end{tabular}

\section{Discussion and Conclusions}

A novel fully automatic segmentation approach for MRA TOF has been shown, especially adapted to the challenging drawback of this method: irregular flow patterns in dedicated regions. By including this knowledge and the segmented vessel skeleton into the level set formulation stable and reliable segmentation results in the carotid bifurcation region have been shown. The achieved segmentation result is an essential basis for simulation of hemodynamics as well as for quantitative measurements of stenosis degree or bifurcation angles which can be used as essential factors in computer aided diagnostics. With this algorithm, the use of standard TOF acquisitions, providing contrast-agent and ionizing radiation free imaging, in the clinical diagnosis and treatment decision workflow becomes feasible. Experimental data including the datasets and ground-truth segmentations are available online at http://www5.cs.fau.de/data/pascal.

Acknowledgements. The authors gratefully acknowledge funding of the Erlangen Graduate School in Advanced Optical Technologies (SAOT) by the German Research Foundation (DFG) in the framework of the German excellence initiative and by Siemens Healthcare.

\section{References}

1. Debrey, S.M., Yu, H., Lynch, J.K., Lövblad, K.-O., Wright, V.L., Janket, S.-J.D., Baird, A.E.: Diagnostic accuracy of magnetic resonance angiography for internal carotid artery disease: a systematic review and meta-analysis. Stroke 39, 2237-2248 (2008)

2. Michaely, H.J., Nael, K.: MRA of the Carotid Arteries. In: Parallel Imaging in Clinical MR Applications, pp. 291-306. Springer, Heidelberg (2007) 
3. Frangi, A.F., Niessen, W.J., Hoogeveen, R.M., van Walsum, T., Viergever, M.A.: Quantitation of Vessel Morphology from 3D MRA. In: Taylor, C., Colchester, A. (eds.) MICCAI 1999. LNCS, vol. 1679, pp. 358-367. Springer, Heidelberg (1999)

4. Gao, X., Uchiyama, Y., Zhou, X., Hara, T., Asano, T., Fujita, H.: A fast and fully automatic method for cerebrovascular segmentation on time-of-flight (TOF) MRA image. J. Digit Imaging 24(4), 609-625 (2011)

5. Ladak, H.M., Milner, J.S., Steinman, D.A.: Rapid three-dimensional segmentation of the carotid bifurcation from serial MR images. J. Biomech. Eng. 122(1), 96-99 (2000)

6. Tang, H., van Onkelen, R.S., van Walsum, T., Hameeteman, R., Schaap, M., Tori, F.L., van den Bouwhuijsen, Q.J.A., Witteman, J.C.M., van der Lugt, A., van Vliet, L.J., Niessen, W.J.: A Semi-automatic Method for Segmentation of the Carotid Bifurcation and Bifurcation Angle Quantification on Black Blood MRA. In: Jiang, T., Navab, N., Pluim, J.P.W., Viergever, M.A. (eds.) MICCAI 2010, Part III. LNCS, vol. 6363, pp. 97-104. Springer, Heidelberg (2010)

7. Watanabe, M., Kikinis, R., Westin, C.F.: Level set-based integration of segmentation and computational fluid dynamics for flow correction in phase contrast angiography. Acad. Radiol. 10(12), 1416-1423 (2003)

8. Yan, P., Kassim, A.A.: MRA Image Segmentation with Capillary Active Contour. In: Duncan, J.S., Gerig, G. (eds.) MICCAI 2005, Part I. LNCS, vol. 3749, pp. 51-58. Springer, Heidelberg (2005)

9. van Bemmel, C.M., Viergever, M.A., Niessen, W.J.: Semiautomatic segmentation and stenosis quantification of $3 \mathrm{D}$ contrast-enhanced $\mathrm{MR}$ angiograms of the internal carotid artery. Magn. Reson. Med. 51(4), 753-760 (2004)

10. El-Baz, A., Farag, A.A., Gimel'farb, G., Hushek, S.G.: Automatic Cerebrovascular Segmentation by Accurate Probabilistic Modeling of TOF-MRA Images. In: Duncan, J.S., Gerig, G. (eds.) MICCAI 2005, Part I. LNCS, vol. 3749, pp. 34-42. Springer, Heidelberg (2005)

11. Scherl, H., Hornegger, J., Prümmer, M., Lell, M.: Semi-automatic level-set based segmentation and stenosis quantification of the internal carotid artery in 3D CTA data sets. Med. Image Anal. 11(1), 21-34 (2007)

12. Suinesiaputra, A., de Koning, P.J.H., Zudilova-Seinstra, E., Reiber, J.H.C., van der Geest, R.J.: Automated quantification of carotid artery stenosis on contrastenhanced MRA data using a deformable vascular tube model. Int. J. Cardiovasc. Imaging (2011)

13. Yushkevich, P.A., Piven, J., Cody Hazlett, H., Gimpel Smith, R., Ho, S., Gee, J.C., Gerig, G.: User-Guided 3D Active Contour Segmentation of Anatomical Structures: Significantly Improved Efficiency and Reliability. Neuroimage 31(3), 1116-1128 (2006)

14. Perktold, K., Peter, R.O., Resch, M., Langs, G.: Pulsatile non-Newtonian blood flow in three-dimensional carotid bifurcation models: a numerical study of flow phenomena under different bifurcation angles. J. Biomed. Eng. 13(6), 507-515 (1991)

15. Marshall, I., Zhao, S., Papathanasopoulou, P., Hoskins, P., Xu, Y.: MRI and CFD studies of pulsatile flow in healthy and stenosed carotid bifurcation models. J. Biomech. 37(5), 679-687 (2004)

16. Li, C., Xu, C., Gui, C., Fox, M.D.: Distance regularized level set evolution and its application to image segmentation. IEEE Trans. Image Process. 19(12), 3243-3254 (2010) 